\title{
Difficulty Analysis for Learners in Problem Solving Process based on the Knowledge Map
}

\author{
Rita Kuo, Wei-Peng Lien ${ }^{1}$, Maiga $\mathrm{Chang}^{2}$ and Jia-Sheng Heh \\ Dept. of Electronic Engineering, Chung-Yuan Christian Univ. \\ ${ }^{1}$ Dept. of Visual Communication Design, Shu-Te University. \\ ${ }^{2}$ Dept. of Special Education, Chung-Yuan Christian Univ.
}

\begin{abstract}
This paper proposes a methodology to calculate both the difficulty of the basic problems and the difficulty of solving a problem. The method to calculate the difficulty of problems is according to the process of building a problem, including Concept Selection, Unknown Designation, and Proposition Construction. Some necessary measures observed in the problem building process are also defined in this paper in order to formulate and calculate the difficulties. Beside the difficulty of the basic problem, four difficulty dimensions, Identification, Elaboration, Planning, and Execution, corresponding to the problem solving steps are also analyzed in this paper. With these difficulty measures learners can understand what kinds of problems they met and what sorts of problem solving strategies are emphasized in solving the problem. To reach our goal, an Item Generating System is constructed for demonstrating and supporting the difficulty calculation in the end of this paper.
\end{abstract}

\section{Knowledge and Problem Analyses}

When suitable knowledge structure is designed, the CAI system can be used to proceed with tutoring, solving problems, or misconceptions diagnosing. The knowledge structure, which used here, is Knowledge Map. Knowledge Map consists of two major parts, Concept Hierarchy and Concept Schema that analyzed by using Knowledge Object. [6][10] Using the Knowledge Map, a problem solving process is constructed as followed. Problem solving has already been discussed for a long while since 1910. [2][3][9] It is a problem when someone has a goal but the goal is blocked for lack of information resources. [5] Two problem structures, Problem Graph and Problem matrix, can be displayed to illustrate this definition. [7]

Based on the previous definition, basic problem architecture has three major parts: Sub-Problem Flag Attribute, Given Attributes and Unknown Attributes, which Sub-Problem Flag Attribute is presented by Core Knowledge Object. Using the problem architecture discussed above, the steps of constructing a problem need three major steps: Concept Selection, Unknown Designation, and Proposition Construction. [7]

\section{Problem Difficulty Features}

Each step of constructing a problem mentioned in previous section decides its difficulty. Ignoring learners' reading ability, this paper only focuses on the first two steps of problem construction. In Concept Selection, number of sub-problems $\left(\gamma_{\text {sub prob }}\right)$ apparently influences the difficulty of the problem. Number of Needed Attributes $\left(\gamma_{\text {need attr }}\right)$ indicates the quantity of attributes needed to learn, which influence the next two features, Learning Sequence (Ylearn_seq) and Concept Depth $\left(\gamma_{\text {cpt_depth }}\right)$. Learning Sequence is a difficulty feature that indicating learning order for learners and Concept Depth shows the specialization of the concept.

The next step, Unknown Setting, affects all attributes described in the problem. Number of Unknown ( $\left.\gamma_{\text {unknown }}\right)$ is one difficulty feature usually used in measuring problem. Number of Given Attribute $\left(\gamma_{\text {given attr }}\right)$ indicates the information this problem supports. Number of Elaborating Attributes ( $\gamma_{\text {elb_attr }}$ ) shows the hidden information cover up in the problem. Attributes setting also influence Mathematical Complexity $\left(\gamma_{\text {math }}\right.$ cpx $)$, which can be another difficulty feature of the problem. This paper focuses on basic problem and disregard knowledge analysis of mathematics. Number of Sub-Problems and Mathematical Complexity will be omitted in the following (article) section.

\section{Difficulty Calculation}

Disregarding the Number of Sub-Problems and Mathematical Complexity features, some needed measures' definitions are listed below and most of them are related to the Knowledge Map.

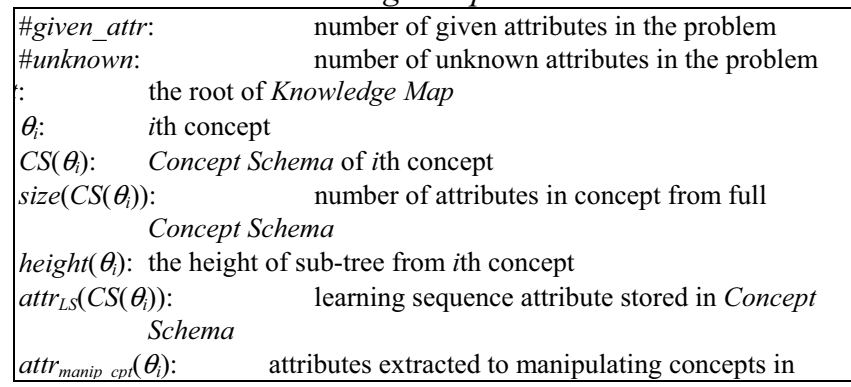




\begin{tabular}{|c|c|}
\hline Problem Ma & \\
\hline $\begin{array}{r}\text { size }\left(\text { attr }_{\text {manip_cpt }}\left(\theta_{i}\right)\right): \\
\text { Matrix }\end{array}$ & number of manipulating concepts in Problem \\
\hline $\operatorname{attr}_{\text {max unknown }}\left(\theta_{i}\right)$ : & maximum number of unknown in $i$ th concept \\
\hline
\end{tabular}

Using these measurement definitions above, each feature of difficulties can be formulized as below:

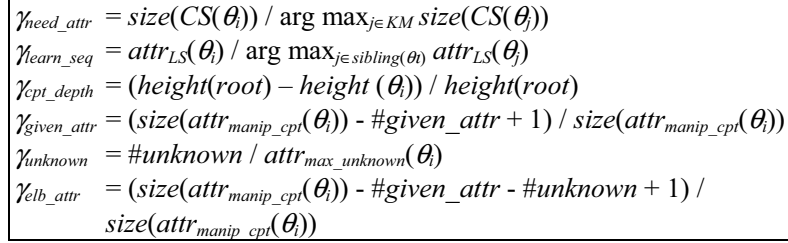

\section{Problem Difficulty Dimensions}

Though the paper analyzes difficulty features corresponding to the problem structure, learners cannot use them easily to understand what ability they get wrong in solving problem. This paper use problem solving analysis to design four types of difficulty for problem solvers realize the characteristics of the problem: Identification Difficulty $\left(\gamma_{i d f}\right)$, Elaboration Difficulty $\left(\gamma_{\text {elb }}\right)$, Planning Difficulty $\left(\gamma_{\text {pln }}\right)$, and Execution Difficulty $\left(\gamma_{\text {exc }}\right)$. These four difficulty dimensions come from the four steps of problem solving in the previous study and all the values of the difficulty dimensions come from the setting of difficulty features. [1][4][6][8][10] To transform difficulty features to difficulty dimensions, the transform formulas are listed below: $\left(\omega_{i j}\right.$ indicates customized weight)

$$
\begin{aligned}
& \gamma_{\text {idf }}=\omega_{21} * \gamma_{\text {learn_seq }}+\omega_{31} * \gamma_{\text {cpt_depth }} \\
& \gamma_{\text {elb }}=\omega_{12} * \gamma_{\text {need_atr }}+\omega_{42} * \gamma_{\text {given_atr }}+\omega_{62} * \gamma_{\text {elb_atr }} \\
& \gamma_{\text {pln }}=\omega_{53} * \gamma_{\text {unnnown }}+\omega_{63} * \gamma_{\text {elb_attr }} \\
& \gamma_{\text {exc }}=\omega_{54} * \gamma_{\text {unknown }}
\end{aligned}
$$

To demonstrate the usage of difficulty dimension, an enhanced Item Generating System based on [7] is constructed. Figure 1 shows an example of generated problem. This problem has one unknown and three given attributes. In the information from Knowledge Map, there are eleven attributes in Concept Schema of "Motion of Constant Acceleration"; seven of them are manipulating concept and three of them are manipulating relations. According to these settings, six difficulty features and four difficulty dimensions are listed below:

$$
\begin{aligned}
& \gamma_{\text {need_attr }}=11 / 13=0.8 \quad \gamma_{\text {given_attr }}=(7-3+1) / 7=0.7 \\
& \gamma_{\text {learn_seq }}=2 / 2=1 \quad \gamma_{\text {unknown }}=1 / 2=0.5 \\
& \gamma_{\text {cpt_depth }}=(4-2) / 4=0.5 \quad \gamma_{\text {eli__atr }}=(7-3-2+1) / 7=0.4 \\
& \gamma_{\text {idf }}=0.5 * 1+0.5 * 0.5=\underline{0.75} \\
& \gamma_{\text {elb }}=0.3 * 0.8+0.3 * 0.7+0.4 * 0.4=\underline{0.61} \\
& \gamma_{\text {ln }}=0.5 * 0.5+0.5 * 0.4=\underline{0.45} \\
& \gamma_{\text {exc }}=1 * 0.5=\underline{0.5}
\end{aligned}
$$

\section{Conclusion and Future Works}

This paper designs an Item Generating System, which constructs four difficulty dimensions for helping learners understanding the emphatic problem solving technique belongs to which problem-solving step. The future work of this paper is to solve the difficulty calculation of complex problem, which influences the Number of SubProblems in difficulty features. Mathematical Complexity also affects the difficulty calculation. It can also become an interesting issue for discussion.

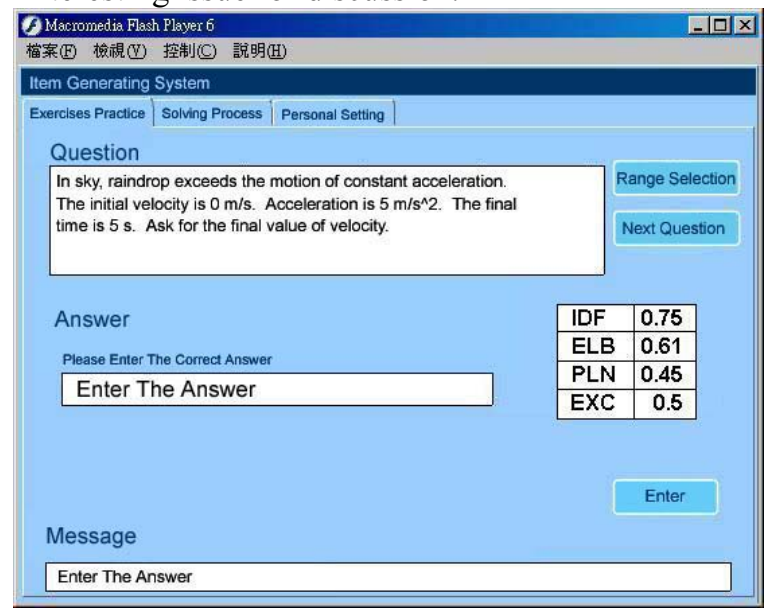

Figure 1. Item Generating System

\section{Reference}

[1] Kun-Fa Cheng, Rita Kuo, Kun-Yuan Yang and JiaSheng Heh (2001), "Intelligent Agent Construction for Problem Solving with Knowledge Schemas," the 17th Workshop on Science Education, Kaohsuang, Taiwan, 2001 (published)

[2] J. Deway, How We Think. Boston, MA: Heath, 1910

[3] F.P. Deek, M. Turoff and J.A. McHugh (1999), "A common model for problem solving and program development," Education, IEEE Transactions on, Volume: 42 Issue: 4, Nov. 1999, pp.331 -336

[4] Chang-Kai Hsu, Rita Kuo, Maiga Chang and Jia-Sheng Heh (2002), "Implementing a Problem Solving System for Physics based on Knowledge Map and Four Steps Problem Solving Strategies," IEEE 2nd International Conference on Advanced Learning Technologies, (ICALT 2002), Kazan, Russia， Sep. 9-12， 2002 (published)

[5] H. Kahney (1993), Problem Solving: Current Issue, $\left(2^{\text {nd }}\right.$ edition) Open University Press, 1993

[6] Rita Kuo, Maiga Chang, Da-Xian Dong, Kun-Yuan Yang and Jia-Sheng Heh (2002), "Applying Knowledge Map to Intelligent Agents in Problem Solving Systems," World Conference on Educational Multimedia, Hypermedia \& Telecommunications (ED-Media 2002), Denver, Colorado, USA, Jun. 24-29, 2002 (accepted)

[7] Rita Kuo, Maiga Chang and Jia-Sheng Heh (2003), "Problem Model Analysis in Inter-Correlated Knowledge by Implementing Item Generating System in Knowledge Map," World Conference on Educational Multimedia, Hypermedia \& Telecommunications (EDMedia 2003), Honolulu, Hawaii, USA, Jun. 23-28, 2003 (accepted)

[8] Sandra P. Marshall (1995), Schemas in Problem Solving, Cambridge University Press, 1995

[9] Polya G. (1965), Mathematical Discovery, New York: Wiley, 1965

[10] Ta-Hsien Tung (2002), "Apply Knowledge Map to Develop Physics Problem-Solving System," Thesis of Master, Dep. Information and Computer Engineering, Chung Yuan Christian University, Chung-Li, Taiwan, R.O.C., Jan, 2002 